IAC-06-B4.2.5

\title{
INTEGRATING INTERNATIONAL ENGINEERING ORGANIZATIONS FOR SUCCESSFUL ISS OPERATIONS
}

\author{
E. Blome \\ International Space Station System Integrator, NASA, USA \\ M. Duggan \\ International Space Station Mission Operations Manager, The Boeing Company, USA \\ L. Patten \\ Columbus Integration, Booz Allen Hamilton, The Netherlands \\ H. Pieterek \\ Head of Operations Support Division, European Space Agency, The Netherlands
}

\begin{abstract}
The International Space Station (ISS) is a multinational orbiting space laboratory that is built in cooperation with 16 nations. The design and sustaining engineering expertise is spread worldwide. As the number of Partners with orbiting elements on the ISS grows, the challenge NASA is facing as the ISS integrator is to ensure that engineering expertise and data are accessible in a timely fashion to ensure ongoing operations and mission success. Integrating international engineering teams requires definition and agreement on common processes and responsibilities, joint training and the emergence of a unique engineering team culture. ISS engineers face daunting logistical and political challenges regarding data sharing requirements. To assure systematic information sharing and anomaly resolution of integrated anomalies, the ISS Partners are developing multi-lateral engineering interface procedures. Data sharing and individual responsibility are key aspects of this plan. This paper describes several examples of successful multilateral anomaly resolution. These successes were used to form the framework of the Partner to Partner engineering interface procedures, and this paper describes those currently documented multilateral engineering processes. Furthermore, it addresses the challenges experienced to date, and the forward work expected in establishing a successful working relationship with Partners as their hardware is launched.
\end{abstract}




\section{INTRODUCTION}

The assembly and operation of the International Space Station (ISS) is a complicated task that requires the integrated effort of organizations from all over the world. The number of space agencies and engineering sites participating in the daily operations of the ISS increases as more hardware is added. Coordinating the work of these multiple sites will become more difficult and more important as the ISS grows.

Anomaly resolution is a particularly vital part of ISS operations and is a key activity of the engineering organizations. The rapid resolution of anomalies and quick return to normal operations is critical to the ongoing success of the ISS program. Because the ISS is a complex system, an anomaly and the steps taken to resolve it can impact other subsystems, even if there are no direct interfaces between the systems. Interactions between subsystems can be unforeseen or unanticipated, so interdisciplinary investigations of anomalies must be thorough and coordinated. And because the safety of the crew or vehicle can be involved, the investigation process must be timely.

Strong, well-defined procedures are a vital foundation for communications and interfaces. Additionally, human interaction is heavily dependent on a set of common perceptions and assumptions that are not necessarily or easily documented. Both of these are a challenge when working in a multi-cultural and multi-lingual environment. This paper first describes Partner to Partner interaction in the early years of the ISS program. Second, the responses to several actual anomalies are discussed to illustrate the gradual evolution and development of a common anomaly resolution approach. And then the new anomaly resolution philosophy that arose from these experiences is explained. In the last section of this paper, three areas of forward work are identified and discussed; the need to complete the interface procedures; the need to create a common engineering culture; the need to define data and information sharing scope and methodologies.

\section{ORIGINAL INTERFACE PROCESSES}

For the first part of the ISS program, anomaly resolution and engineering interface processes developed from a mixture of processes from previous programs and ad hoc procedures instigated on the spot. A single multi-lateral engineering process did not exist.

The first on-orbit operational interfaces and consequently engineering interfaces were between Russia and U.S.A in 1998 after their modules were launched and mated. This was followed by the launch and operations of the Canadian contribution in 2001.

The U.S. and Russia came to the ISS program without a long history of working together, although some lessons were learned through their joint Shuttle-MIR missions. However, both Partners had a long history of human space flight and well established procedures for its execution. Both Partners initially implemented their traditional human space flight methods in the ISS. 
The initial engineering interactions can be characterized by difficult communication and inefficient interfaces. Several factors contributed to these initial communication challenges; the initial contacts and interfaces were primarily limited to the management level which did not adequately communicate down to their respective engineering teams; lack of jointly available communication tools; difference in methods and culture and the lack of familiarity of the assumptions and perceptions between teams.

The initial environment, characterized by difficult communication and inefficient interfaces, resulted in very challenging integrated anomaly resolution. Direct communication between U.S. and Russian engineers was difficult to set up. There were no facilities or methods to easily share engineering data. No shared agreement existed, formal or otherwise, on how to jointly resolve anomalies. The eventual joint decisions and agreements often had to go to the highest management levels for approval, an inefficiency that prolonged the process.

In the U.S./Russian situation, the problem that the ISS program faced was the need to establish interfaces at the lowest levels to share information and an agreement on a common process to resolve anomalies.

Over the first several years of on-orbit operations, ISS issues and daily activities made both Partners realize that cooperation and communication at all levels was important to ensure smooth operation of the ISS. They also gained more trust and understanding of each other. The desire and culture for integrated anomaly resolution grew. U.S. and Russian engineering counterparts started meeting more frequently and exchanging greater amounts of information. In particular, the environmental discipline engineering teams were driven together by the need to manage the shared ISS cabin atmosphere. The two Partners also became more familiar with each other's processes. A common understanding and culture was developing. These shared understandings and the realization of their importance led to the desire to establish and formalize formal multi-lateral engineering interfaces.

The U.S. and Canada have a long history of working together on the Space Shuttle program and are very familiar with each other and in many ways the ISS program was a continuation of that relationship. There is a significant Canadian presence in the NASA mission control center, which means that many discussions and interactions can be face to face. The extended joint operational history and the collocation of personnel resulted in a very efficient engineering interface process between the U.S. and Canada and the emergence of a single joint process that is employed by both, but driven by NASA's operational experience.

Cooperation between U.S. and Canadian engineers can be so close that the distinction between U.S. and Canadian engineers might not be apparent to an outside observer. Both meet regularly and have access to common mission execution and anomaly databases. While this close U.S./Canadian relationship has the benefit of quick and efficient cooperation for anomaly resolution, the lack of formal process definition has meant that sometimes decisions and conclusions were not elevated to the proper management levels for review and concurrence. Therefore one issue that emerged from this successful cooperation was the need to formalize the 
coordination and approval processes that respected the prerogatives of each Partner. This was eventually addressed in the development of formal multi-lateral engineering interfaces.

\section{ANOMALY RESOLUTION EXAMPLES}

Many ISS onboard anomalies requiring an integrated response have been successfully resolved. These provide excellent examples of the integrated engineering interactions that must occur to resolve anomalies. Some examples illustrate the unexpected interactions and unintended consequences that can occur between subsystems. Others demonstrate the importance of keeping other engineering organizations informed of the progress of seemingly non-integrated anomaly resolution. An example of coordinated anomaly resolution leading to an efficient and correct result is also discussed.

\section{Example 1-Failed Power Distribution $\underline{\text { Box }}$}

A power distribution box in the US segment of ISS automatically turned off ('tripped off') as a self-regulated response to a problem. This box provides power to a power converter in the Russian segment. The trip created an immediate cross-partner impact because power suddenly was no longer being provided to the Russian segment. Because of the history of this type of power distribution box, both American and Russian engineers initially believed that this trip was a reoccurrence of a previously experienced problem with this particular design. American engineers continued to investigate in order to confirm this hypothesis and Russian engineers undertook no further investigation in the Russian segment. However, the American investigation eventually determined that the trip was the result of an external overcurrent event and that the American power distribution box had responded nominally to an unsafe external current level. Russian engineers resumed their investigation and subsequently determined that an internal short in the Russian power converter was the cause of the overcurrent.

In this case, the integrated impact of the anomaly was immediately apparent to all parties. Though both American and Russian engineers initially assumed an incorrect cause of the anomaly and ceased integrated anomaly resolution, the exoneration of the American hardware led to the resumption of communication and integrated anomaly resolution.

\section{Example 2-High Humidity Levels in Plant Growth Payload}

During the operation of a plant growth payload in the US segment, humidity levels within the payload rose to levels that threatened the success of the experiment. Payload engineers launched an investigation that concluded that an airflow restriction caused by a noise muffler was the cause of the high humidity. The payload engineers asked the crew to remove the muffler and humidity levels began to decrease. Learning of the muffler removal through their regular review of payload anomaly reports, program safety engineers subsequently questioned the impact of the muffler removal on noise levels in the US segment. Program safety and systems engineers then started a second investigation of the situation. Acoustic measurements confirmed that onboard sound levels had increased and the crew confirmed that the levels, while tolerable, were annoying. Systems engineers were able to adjust the ISS thermal system settings in a 
way that controlled the humidity levels in the payload and allowed the muffler to be reinstalled.

This example illustrates the potential pitfall of uncoordinated anomaly resolution: unintended consequences caused by a lack of communication. The payload engineers did not foresee the effect of the first solution to the problem, an increase in noise levels due to the removal of the muffler. Furthermore, the payload and systems engineers lacked an effective and established method to cooperate and collaborate.

Coordination and communication between all participants, even those not known to be affected, may result in solutions that provide greater risk reduction and increase anomaly resolution effectiveness. This particular anomaly led to review and improvement of the interface procedures between the US payloads and systems engineers.

\section{Example 3-Russian Oxygen Generation System}

The Russian oxygen generation system started experiencing periodic shutdowns and the Russian team started troubleshooting. Because oxygen generation and consumption is a basic requirement for continued ISS operation, the U.S. engineering team closely monitored the anomaly resolution process. This system electrolyzes water to generate oxygen and generates hydrogen as a by-product. The hydrogen is normally vented overboard and the oxygen output into the ISS is monitored for hydrogen by a gas analyzer. As part of the troubleshooting and recovery, the gas analyzer was disabled. Knowing the importance of this gas analyzer and that it is an important hazard control, the U.S. and Russian safety teams worked together throughout the troubleshooting to analyze the configuration and assure that adequate hazard controls were maintained. Based on this coordination, additional monitoring was implemented to maintain the required hazard controls.

This was an excellent example of integrated anomaly resolution. The Russian team completely handled the engineering troubleshooting of the problem, which was not an integrated issue. Control of the integrated hazard, inadvertent release of hydrogen into the ISS cabin, is an integrated issue and the U.S. and Russian teams cooperated to keep each other informed and implement appropriate safety precautions.

\section{NEW PARTNER TO PARTNER INTERFACE PROCESSES}

With the launch of hardware from other Partners on the horizon, the ISS Program started a new effort to document and formalize engineering procedures. These procedures build on previous experience and incorporate lessons learned.

The ISS experience with anomaly resolution and the gradual evolution and development of integrated anomaly resolution processes led to two key realizations. First, the engineers who designed and built a system will always understand the system best within the constraints of ongoing ISS operations. This implied that while other Partners' engineers might be asked to understand or validate certain aspects of the system, responsibility for the operation, maintenance and anomaly resolution of those systems should be left with the design/development engineers. The second 
realization was that in the complex systems environment of the ISS, integrated systems affects and impacts were not always apparent and a Partner could easily affect another Partner through their actions without being aware of it. This realization implied that a certain amount of integrated information about system operations and anomalies must always be shared between Partners even when the potential impact is not readily apparent. These two realizations became the guiding factors in the negotiation and development of the multilateral engineering interface procedures.

Through multilateral negotiations, agreements are being made to define how the ISS Partners will communicate engineering issues. Each Partner is responsible for managing engineering support and analysis for their specific systems and for identifying, documenting, and resolving anomalies involving their hardware and software in accordance with their own processes. Each Partner is required to share a certain level of information to allow other Partners to determine the potential effects on their systems. And NASA, as the overall integrator, must be aware of the issues being worked, and is required to ensure that information from one Partner that affects other Partners is appropriately disseminated.

Standing technical bilateral and multilateral forums have been created for discussing both on-going nominal operation of the ISS systems and resolution of anomalies. The forums exchange engineering data as necessary to ensure the safe and continued operation of ISS. These forums are under the direction of the each Partner's subsystem managers and meet as needed.
Integrated anomaly resolution presents a unique challenge to the ISS. Distance, experience, culture, and languages separate teams. Thus, extensive work is being done to ensure agreements are in place prior to the launch of European Space Agency (ESA) and Japan Aerospace Exploration Agency (JAXA) elements. These agreements build on the experience to date in ISS anomaly resolution.

Special recognition is also given to the importance of real-time anomaly resolution in a human space flight program. When an anomaly occurs, it is of utmost importance that any actions necessary to place the ISS and its crew in a safe configuration are taken immediately. After that, the next goal is to implement corrective actions that allow the safe and continued operations of the ISS for an extended period of time. While each Partner is responsible for determining the root cause of a failure in their hardware, the new processes recognize the importance of establishing a multilateral forum that will be used to discuss the impacts of the failure.

To date, these procedures have been defined conceptually in a generic document. Most importantly, agreements have been made stating each Partner is responsible:

For participating in the appropriate bilateral and multilateral engineering forums to discuss integrated anomalies.

For investigating, documenting and closing anomalies involving their hardware and software in accordance with their own processes.

\section{FORWARD WORK}

In order to successfully integrate international engineering organizations for successful ISS operations, there are three 
areas of work that must be completed. First, the engineering interface procedures must be completed, tested and refined. Second, the data and information exchange agreements and methodologies must be established. Third, a common international ISS engineering team culture must emerge.

\section{Completion of Integrated Procedures}

The generic procedures for sustaining engineering have been under development since 2004 and a basic version has been published. While the multi-lateral engineering philosophy will be common to all of the ISS Partners, it is recognized that there will be differences in specific implementation for each Partner. These differences are captured in annexes to the multi-lateral engineering interface document. To date, the only annex that is under development is the European Space Agency (ESA) Automated Transfer Vehicle annex. Work is scheduled to begin shortly for annexes regarding the Japanese Aerospace Exploration Agency (JAXA) Japanese Experiment Module and the ESA Columbus module.

Prior to the launch of ESA's or JAXA's modules, it is hoped that multilateral Partner engineering team simulations and training sessions will be held for operations specific to each module. The goal being to gain a better understanding of each Partners' processes and to exercise the multi-lateral interface and anomaly resolution procedures. Early participation from ESA and JAXA engineering teams in operations preparation activities such as simulations will provide a large benefit in terms of training and building a common understanding. This will enable the ISS program to test and refine and further develop the interface procedures.

\section{Data and Information Sharing}

Data and information sharing on a day to day basis in the ISS program still has to be clearly defined. The exchange of information and technical data has two major hurdles at present. First, industrial policy and privacy issues must be respected since the hardware providers are primarily commercial companies. Second, US export control regulations add a significant overhead and restriction to data and information sharing.

In an industrial policy driven world it is not sufficient to state that 'required data will be shared'. Companies building and providing hardware have an interest in retaining their own intellectual property. Therefore, the interface between Partners must be cooperative to resolve anomalies at an integrated level but restricted to protect a companies intellectual property. Therefore, for example, root cause analysis data cannot be provided to the ISS Program level.

Following the concepts agreed to via ISS Program Memorandum of Understandings at the government level and implementation at the National Space Agency levels, the scope of the Partner data sharing requirements are defined. These agreements must be respected in any interface procedure data sharing agreements.

NASA has experienced difficulties in providing required data and personnel to international Partners due to the U.S. export control regulations. This has particularly hindered timely information exchange and has a potential to impact the integrity of the engineering interface procedures for anomaly resolution. Therefore, NASA must work to achieve a solution that follows U.S. 
export control regulations while assuring ISS crew and vehicle safety.

Assuming all data and information sharing agreements are resolved, the next issue to conquer is the means through which this data will be exchanged and a common data exchange format. Adaptation of existing tools will be required in order to easily exchange information between the Partners. NASA plans to expand its existing tools and provide additional access for Partners. ESA has already started development on an internal anomaly tracking system.

\section{Common Culture}

Developing a common culture for a truly integrated multi-lateral engineering team that is spread around the globe will be a challenge. Developing a common culture and a common attitude is an important part of team development. Experience has shown that an important factor in developing the culture of the U.S. engineering team is spending time together. By working and spending much time together, team members develop a common outlook.

As seen from the initial joint operations between Russia and the U.S., a common culture will emerge once it is necessitated through common experience. Although in the case of US and Russian this common experience was only realized through onorbit operations, there is a potential that early involvement in ESA and JAXA operations preparation activities will improve this timeline.

A mutual respect and understanding of each Partners operations approach and methodology is required before a single culture will emerge. Additionally, all teams will have to recognize that the emergent engineering culture will be a unique engineering culture rather than the adoption of a single dominant culture.

\section{CONCLUSIONS}

The U.S., Canadian, and Russian Partners are cooperating well today. The ISS program is poised to integrate JAXA and ESA into the daily on-orbit operations of the program. Since initial joint ISS operations have started the multi-lateral engineering interfaces and anomaly resolution have advanced considerably.

Initial international interfaces were based on improvised procedures and good intentions. Now procedures are being developed that should provide a robust, effective and efficient anomaly resolution process and engineering interface. Considerable gains in cooperation were realized simply through time spent working together. A key advance in this development is formalization of the philosophy that a certain level of data must be shared freely between Partners while maintaining respect for each Partner's engineering expertise and prerogative. This is a true challenge in today's political and commercial environment. 